\title{
Longitudinal measurements of syrinx size in a rat model of posttraumatic syringomyelia
}

\author{
Elmira Najafi, MBBS, ${ }^{1}$ Lynne E. Bilston, PhD, ${ }^{2,3}$ Xin Song, MMed, ${ }^{1}$ Andre Bongers, PhD, ${ }^{5}$ \\ Marcus A Stoodley, PhD, ${ }^{1}$ Shaokoon Cheng, PhD, ${ }^{4}$ and Sarah J. Hemley, PhD ${ }^{1}$ \\ ${ }^{1}$ Faculty of Medicine and Health Sciences and ${ }^{4}$ Faculty of Science and Engineering, Macquarie University, ${ }^{2}$ Neuroscience \\ Research Australia, and ${ }^{5}$ Lowy Cancer Research Centre, University of New South Wales, Sydney; and ${ }^{3}$ Prince of Wales Clinical \\ School, University of New South Wales Medicine, Randwick, New South Wales, Australia
}

\begin{abstract}
OBJECTIVE Syringomyelia pathophysiology is commonly studied using rodent models. However, in vivo studies of posttraumatic syringomyelia have been limited by the size of animals and lack of reliable noninvasive evaluation techniques. Imaging the rat spinal cord is particularly challenging because the spinal cord diameter is approximately 1-3 $\mathrm{mm}$, and pathological lesions within the spinal cord parenchyma are even smaller. The standard technique has been histological evaluation, but this has its limitations. The aim of the present study was to determine whether syrinx size could be reliably measured using a preclinical high-field MRI animal system in a rat model of posttraumatic syringomyelia.
\end{abstract}

METHODS The authors used an existing rat model of posttraumatic syringomyelia, which was created using a controlled pneumatic compression device to produce the initial spinal cord injury, followed by a subarachnoid injection of kaolin to produce arachnoiditis. T2-weighted MRI was performed on each animal using a 9.4-T scanner at 7, 10, and 13 weeks after injury. Animals were killed and syrinx sizes were calculated from in vivo MRI and histological studies.

RESULTS MRI measurements of syrinx volume and length were closely correlated to histological measurements across all time points (Pearson product moment correlation coefficient $r= \pm 0.93$ and 0.79 , respectively).

CONCLUSIONS This study demonstrates that high-field T2-weighted MRI can be used to measure syrinx size, and data correlate well with syrinx size measured using histological methods. Preclinical MRI may be a valuable noninvasive technique for tracking syrinx formation and enlargement in animal models of syringomyelia.

http://thejns.org/doi/abs/10.3171/2015.10.SPINE15538

KEY WORDS MRI; syrinx; posttraumatic syringomyelia; spinal cord injury

$\mathrm{P}$ OSTTRAUMATIC syringomyelia is a serious disorder characterized by the development of fluid-filled cavities within the spinal cord. A retrospective study of patients with spinal cord injury reported that $7.3 \%$ developed posttraumatic syringomyelia within 5 years of the initial injury, ${ }^{17}$ and this number would presumably increase with time. As a syrinx expands, it causes damage to the surrounding spinal cord tissue and may result in pain or progressive loss of sensory or motor function. MRI is the gold standard for imaging acute spinal cord injury, with sagittal T2-weighted sequences providing the most useful information, ${ }^{5}$ and it is the most commonly used tool for diagnosing syringomyelia in these patients. ${ }^{1}, 10,11,15,19,22$

In animal studies, posttraumatic syringomyelia has tra- ditionally been assessed using histological methods.-9,16 These studies have been extremely valuable in adding to our knowledge of the condition; however, histology offers only a snapshot of a syrinx cavity. It does not allow the progression and expansion of the syrinx to be visualized. Given that the diameter of the rat spinal cord is approximately $1-3 \mathrm{~mm}^{3}$ and pathological lesions in the spinal cord are even smaller, studying these lesions noninvasively proves challenging.

A limited number of studies have used different imaging techniques to study posttraumatic syringomyelia. Schwartz et al. used diffusion-weighted MRI with apparent diffusion coefficient (ADC) maps to investigate spinal cord cavities in rats following injection of an excitotoxic 
amino acid into the spinal cord gray matter. ${ }^{21}$ They found that areas of intense signal on ADC maps correlated with cavities evident on histological sections filled with cellular debris, and both T1- and T2-weighted MR images and ADC maps showed a region of changed signal intensity in the central cord that corresponded to a cyst. ${ }^{21}$ Another study of posttraumatic syringomyelia used T2-weighted images to identify a syrinx in rats prior to lumboperitoneal shunt insertion but was not of adequate resolution to determine lesion length. ${ }^{8}$ To the best of our knowledge, this is the first study to use high-field MRI to calculate lesion volume in a rat model of posttraumatic syringomyelia.

Preclinical high-field MRI has the potential to noninvasively monitor and track pathological changes in the spinal cord during syrinx development, but the feasibility and accuracy of MRI-based measurements of syrinx size have not been studied. The aim of this study was to evaluate the utility of high-field MRI in tracking the development and enlargement of syrinxes in a rodent model of syringomyelia.

\section{Methods}

This study was approved by the animal care and ethics committees of Macquarie University and the University of New South Wales. A previously developed rat model of posttraumatic syringomyelia was used. ${ }^{24} \mathrm{~A}$ total of 20 male Sprague-Dawley rats weighing 200-250 g and aged 4-5 weeks were used in this study. Groups consisted of 15 animals that underwent the syrinx induction procedure (described below) and 5 normal control animals. Animals were scanned at 7, 10, and 13 weeks after syrinx induction surgery. At each time point, a subset of animals in each group was killed, and histological comparison was made with the MRI measurements (Table 1). In vivo MRI measurements were supplemented with higher-resolution, ex vivo MRI measurements on fixed spinal cord samples.

\section{Syrinx Induction}

The posttraumatic model of syringomyelia was created using a controlled pneumatic compression device (The Infinite Horizon Impactor; Precision Systems and Instrumentation) to produce an initial spinal cord injury, followed by a subarachnoid injection of kaolin. All procedures were performed in a sterile field under general anesthesia induced with $5 \%$ isoflurane in oxygen $(1 \mathrm{~L} / \mathrm{min})$ and maintained with $2 \%-2.5 \%$ isoflurane through a nose cone. Animals were placed prone, and the skin was shaved and prepared with povidone iodine. Guided by the spinous process of $\mathrm{T}-2$ in the midline cervicothoracic junction, an incision was made over the cervicothoracic junction, and
C7-T1 laminectomies were performed. The spinous processes of C-6 and T-2 were clamped and stabilized using a stereotactic device, and the spinal cord impactor was positioned over the cord with the 2-mm impactor tip just touching the dural surface.

The spinal cord impactor was calibrated to deliver 75 $\mathrm{kDyn}$ to the spinal cord, chosen to produce posttraumatic syringomyelia without producing significant paralysis or neurological deficits in animals. ${ }^{24}$ A $5-\mu l$ suspension of $250 \mathrm{mg} / \mathrm{ml}$ kaolin in sterile saline was then injected into the subarachnoid space (Sigma-Aldrich). The wound was closed with a 4-0 absorbable Vicryl suture. At the conclusion of the operation, $0.05 \mathrm{mg} / \mathrm{kg}$ of $324 \mu \mathrm{g} / \mathrm{ml}$ buprenorphine was administered subcutaneously. Subsequent doses were given as needed. The animals were allowed food and water ad libitum and monitored for any excessive weight loss, limb weakness, or signs of excessive self-grooming.

\section{In Vivo MRI}

In vivo imaging was performed using a Bruker Biospec 9.4-Tesla MRI scanner (model 94/20 USR; Bruker Corp.) in the animal high-field MRI facility at the Biological Resources Imaging Laboratory at the University of New South Wales. A circular, polarized, whole-body radiofrequency, 72-mm-volume quadrature coil was used for radiofrequency transmission and reception. T2-weighted MRI was performed at multiple time points to chart the progression of syringomyelia. During in vivo MRI procedures, general anesthesia was induced in an induction chamber with $5 \%$ isoflurane in oxygen $(1 \mathrm{~L} / \mathrm{min})$ and maintained with $2 \%-2.5 \%$ isoflurane through a nose cone. The animals were placed prone on the animal bed frame with the head stabilized with a tooth bar and the spinal cord lesion approximately centered within the receiving coil. Respiration and heart rate were monitored during the imaging procedures. Animals' body temperature was maintained by a temperature-controlled warming blanket set at $36^{\circ} \mathrm{C}$.

Sagittal and axial T2-weighted images were acquired using a rapid acquisition with relaxation enhancement (turbo RARE) method. Sequence parameters for sagittal acquisition were as follows: TE (eff) $26 \mathrm{msec}$, TR 2200 msec, slice thickness $0.7 \mathrm{~mm}$, slice gap $0.3 \mathrm{~mm}$, field of view (FOV) $50 \times 30 \mathrm{~mm}$, acquisition matrix $284 \times 384$ $\mathrm{mm}$, in-plane resolution $130 \times 130 \mu \mathrm{m}$, and 3 signal averages. Sequence parameters for axial acquisition were as follows: TE $18 \mathrm{msec}$, TR $2000 \mathrm{msec}$, slice thickness 0.7 $\mathrm{mm}$, slice gap $0.9 \mathrm{~mm}$, FOV $20 \times 20 \mathrm{~mm}$, acquisition matrix $150 \times 150$, in-plane resolution $133 \times 133 \mu \mathrm{m}$, and 18 signal averages. The duration of each MRI acquisition was approximately 30 minutes.

At each time point after scanning, the animals selected

TABLE 1. Experimental and control groups

\begin{tabular}{|c|c|c|c|c|c|c|}
\hline \multirow[b]{3}{*}{ Imaging Method } & \multicolumn{6}{|c|}{ No. of Animals at Each Time Point } \\
\hline & \multicolumn{2}{|c|}{7 Weeks } & \multicolumn{2}{|c|}{10 Weeks } & \multicolumn{2}{|c|}{13 Weeks } \\
\hline & Control & Syrinx & Control & Syrinx & Control & Syrinx \\
\hline In vivo MRI & 5 & 15 & 4 & 10 & 2 & 5 \\
\hline Ex vivo MRI & 1 & 5 & 2 & 5 & 2 & 5 \\
\hline Histology & 1 & 5 & 2 & 5 & 2 & 5 \\
\hline
\end{tabular}


for histological examination were anesthetized and rapidly killed by intracardiac perfusion of 1250 IU heparin in 250 $\mathrm{ml}$ of cold phosphate-buffered saline followed by $250-350$ $\mathrm{ml}$ of $4 \%$ paraformaldehyde (Lancaster Synthesis) in 0.1 M phosphate buffer (pH 7.4) at a flow rate of $50 \mathrm{ml} / \mathrm{min}$.

\section{Ex Vivo MRI}

Following intracardiac perfusion, each spinal cord was postfixed with $4 \%$ paraformaldehyde overnight at $4^{\circ} \mathrm{C}$. The whole spinal cord was then extracted and fitted into a glass Pasteur pipette sealed at both ends and filled with phosphate-buffered saline. Bundles of 3 tubes were placed in the MR scanner. Spinal cords were aligned within each tube such that the location of the syrinx was consistent across all specimens. A circular polarized, 15-mm-volume, radiofrequency quadrature sample coil was used for radiofrequency excitation/reception. High-resolution, T2weighted images were acquired using a 3D turbo RARE sequence with the following parameters: TE $50 \mathrm{msec}$, TR $2000 \mathrm{msec}$, slice thickness $0.15 \mathrm{~mm}$, FOV $15 \times 15 \times 15$ $\mathrm{mm}$, acquisition matrix $230 \times 230 \times 100$, resolution $65 \times$ $65 \times 150 \mu \mathrm{m}, 50 \%$ oversampling in phase-encoding z-direction, and 3 signal averages. The duration of each MRI acquisition was approximately 3.5 hours. The ex vivo MR images were used to create a $3 \mathrm{D}$ model of the spinal cord using a 3D slicer. ${ }^{13}$

\section{Histological Examination}

After ex vivo MRI, each spinal cord was cryoprotected in $30 \%$ sucrose in phosphate buffer at $4^{\circ} \mathrm{C}$ for a minimum of 24 hours. A segment of spinal cord from C-5 to T-2 was then embedded in optimum cutting temperature compound (ProSciTech). Transverse $20-\mu \mathrm{m}$ sections of spinal cord containing the syrinx cavity were cut on a cryostat (Leica CM 1950 cryostat; Leica Biosystems Nussloch). For each animal, consecutive sections were taken from C-5 to T-2 to ensure the entire syrinx cavity was collected for histological analysis. Sections were stained with $\mathrm{H} \& \mathrm{E}$ for histological comparison. Images were obtained using a digital camera (Zeiss Z1; Carl Zeiss AG), and the size of the syrinx was calculated using ImageJ software (National Institutes of Health). The spinal cord in each histological section was manually traced to calculate the whole spinal cord area, and automatic thresholding was applied to delineate the syrinx and calculate the syrinx area in $\mu \mathrm{m}^{2}$. The volume of the syrinx was calculated for each tissue slice, and the total volume of the syrinx was determined by summing the volumes for each consecutive tissue section. Relative volume (\%) of the syrinx was calculated as a percentage of the overall spinal cord volume for the corresponding spinal segment. The maximum length of the syrinx cavity was also estimated by counting the number of sections containing syrinx and multiplying this number by $20 \mu \mathrm{m}$, the thickness of each section.

\section{MR Image Analysis}

Axial MR images were used to quantify the syrinx volume. All MR images were analyzed using the ImageJ image-processing toolkit. The spinal cord was manually traced to calculate total spinal cord area in each section.
The mean gray values of common anatomical structures were used to subtract background (mean gray values from airway) and normalize each MRI slice (mean gray values of muscle). An appropriate threshold (manually chosen) was applied to all images to delineate the syrinx (hyperintense signal) from the gray and white matter. Once the area of the syrinx and whole spinal cord cross-section was determined in each section, the pixel value for each MRI slice was used to calculate the syrinx volume in $\mathrm{mm}^{3}$. Lesion volume was calculated by multiplying total lesion pixel count with individual voxel volume. Syrinx volume was then presented as a percentage of the whole spinal cord volume for the corresponding segment of cord. The maximum length of the syrinx cavity was measured on sagittal images using RadiAnt DICOM Viewer (Medixant).

\section{Statistical Analysis}

A paired t-test with Bonferroni adjustment was used to compare syrinx volumes from MR images and histological sections. A 1-way ANOVA with repeated measures or multiple comparisons was used to compare syrinx volumes calculated from MRI in individual animals at different time points. A p value $<0.05$ was considered statistically significant. The Pearson correlation coefficient (r) was calculated to evaluate the relationship between syrinx volume and length from MRI and histology using Prism 6 (GraphPad Software).

\section{Results}

All animals survived the surgical procedures without significant weakness or neurological deficits, although self-injurious neuropathic scratching was observed in 7 of the 15 experimental animals. ${ }^{6}$ All experimental animals developed a syrinx (syrinx animals). No control animals developed a syrinx or spinal cord edema.

\section{Histological Observations}

Histological assessment indicated progressive pathological changes in the injured spinal cord over the 13 weeks after syrinx induction, including edema, hemorrhage, inflammation, astrocyte proliferation, progressive cavitation at the site of injury, and rostrocaudal degeneration. After 7 weeks, edema, hemorrhage, inflammation, and astrocyte proliferation were common, and a syrinx began to form (Fig. 1H-J). Evidence of low-density tissue within the cavities was present (Fig. 1E-J). The mean rostrocaudal length $( \pm \mathrm{SD})$ of the syrinx at this time point was $2.2 \pm 0.40$ $\mathrm{mm}(\mathrm{n}=5)$ (Table 2). Frank syrinx formation and enlargement was observed at 10 weeks. A high-density astroglial scar adjacent to the syrinx was observed at 10 weeks, as well as multiple cavities, which were separated by septa. An enlarged, intact central canal was observed in most (4 of 5) of the spinal cords (Fig. 2A-C). The mean rostrocaudal syrinx length at 10 weeks was $3.5 \pm 1.0 \mathrm{~mm}$ (Table 2). By 13 weeks after syrinx induction, evidence of astroglial proliferation was observed adjacent to the syrinx cavities and in the septa between multiple cavities. Large, welldefined, multilocular cavities and some evidence of lowdensity tissue surrounding the cavities was observed (Fig. 

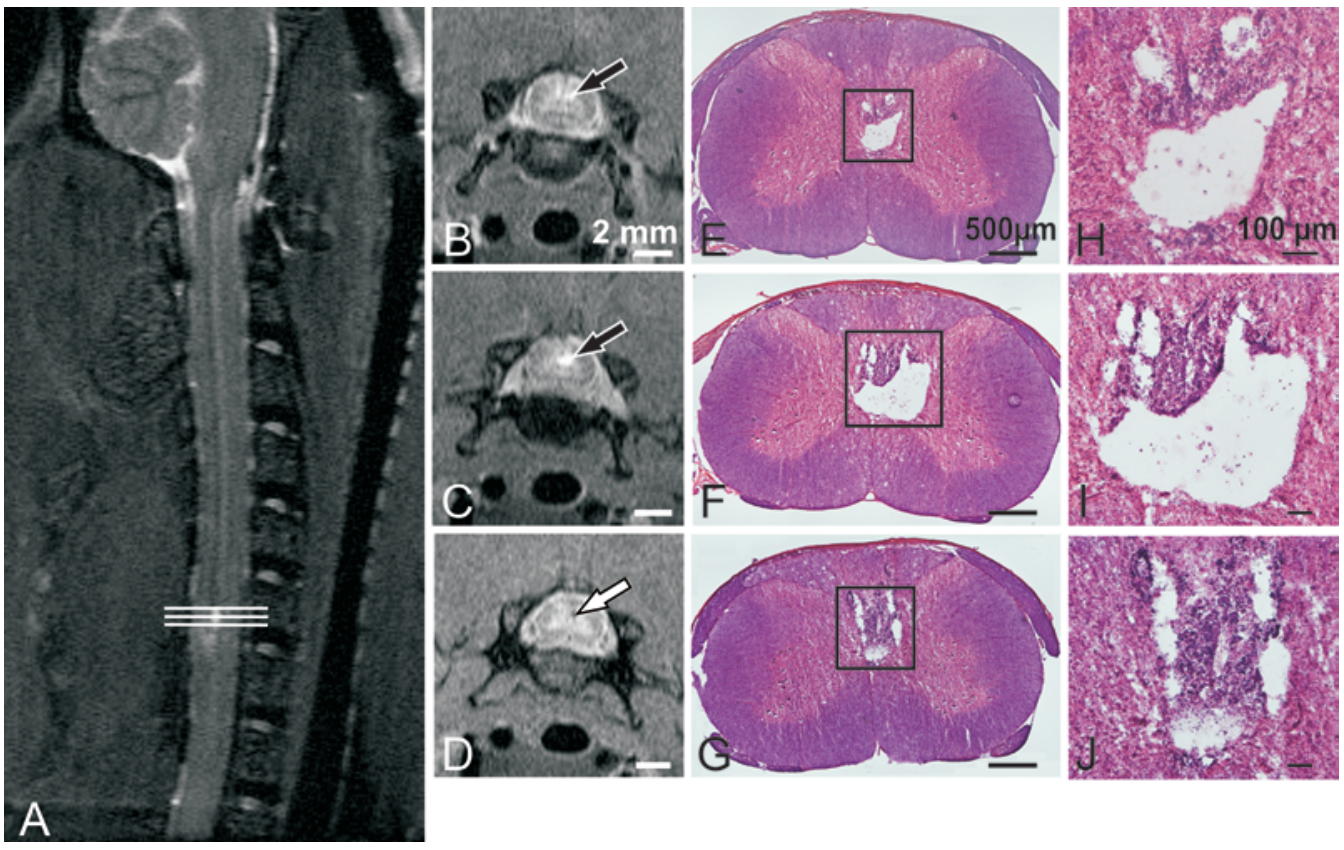

FIG. 1. Correlation between T2-weighted MRI and histological changes in a rat 7 weeks after syrinx induction. Sagittal (A) and axial (B-D) views showing the syrinx (black arrow, B and C) and a hypointense region (white arrow, D). E-G: Histopathological counterparts of the in vivo MR images. H-J: High-magnification images of syrinx cavity. Figure is available in color online only.

$3 \mathrm{~A}-\mathrm{C})$. The mean rostrocaudal syrinx length at 13 weeks was $4.0 \pm 1.6 \mathrm{~mm}$ (Table 2), extending over a maximum of 2 spinal levels. There was a progressive increase in syrinx length with time after syrinx induction; however, this did not reach statistical significance (1-way ANOVA, $\mathrm{p}=$ 0.059 ) (Table 2). Syrinx volume increased from 7 weeks to 10 and 13 weeks but did not reach statistical significance (1-way ANOVA, $\mathrm{p}=0.12$ ).

\section{MRI}

MRI yielded high-resolution axial images of the rat cervical and thoracic cord without significant motion artifacts. In control animals, the gray matter was more hyperintense than the surrounding white matter, and the typical butterfly shape of the gray matter was clearly defined. The CSF in the subarachnoid space could be distinguished as a hyperintense band surrounding the spinal cord (Fig. 4A and C).

In syrinx animals, distinct hyperintense regions (syrinx) were observed in both axial and sagittal images (Fig. 4B and D). On sagittal images, the site of laminectomy was evident by the absence of 2 spinous processes (Fig. 4D). In most (10 of 15) animals, a well-defined syrinx could be seen at each time point, although, at 7 weeks, the hyperintense region was diffuse in some animals (4 of 15) rather than the well-delineated areas observed consistently at 10 and 13 weeks. Axial MR images indicated animals with multiloculated cavities (Fig. 3F, J, and N). The CSF in the subarachnoid space was visible as a thin hyperintense region around the spinal cord except where kaolin was present. Gray matter and white matter were not always easily differentiated, because the fluid-filled cavity was hyperintense compared with the surrounding spinal tissue, and the gray matter in some slices was largely occupied by the syrinx. In all syrinx animals, axial sections revealed a small area of signal intensity loss in the central gray or white matter of the cord or in close proximity to the syrinx (Fig. 1D). This corresponded to sites of hemorrhage and the presence of hemosiderin deposits in the histological sections (Fig. 1G and J).

In animals that had serial MR images, the hyperintense syrinx volume progressively increased in size from 7 weeks to 13 weeks, but these changes did not reach statistical significance (1-way ANOVA, $p=0.15$ ). The location and shape of the syrinx in the in vivo MR images closely matched the corresponding histological sections (Fig. 2).

On axial MR images of fixed ex vivo spinal cords, gray matter was also clearly distinguishable from the less-intense white matter, showing greater contrast than the in

TABLE 2. Syrinx size calculated from histological sections and MR images

\begin{tabular}{llcc}
\hline \multicolumn{1}{c}{ Parameter } & 7 Weeks & 10 Weeks & 13 Weeks \\
\hline Syrinx length from histology $(\mathrm{mm})$ & $2.2 \pm 0.40$ & $3.5 \pm 1.0$ & $4.0 \pm 1.6$ \\
\hline Syrinx length from MRI $(\mathrm{mm})$ & $2.1 \pm 0.6$ & $2.9 \pm 1.1$ & $3.0 \pm 0.9$ \\
\hline Syrinx volume from histology* & $2.2 \% \pm 0.65 \%$ & $6.7 \% \pm 5.8 \%$ & $8.2 \% \pm 3.6 \%$ \\
\hline Syrinx volume from MRI* $^{*}$ & $2.8 \% \pm 1.9 \%$ & $8.9 \% \pm 8.7 \%$ & $11 \% \pm 3.8 \%$ \\
\hline
\end{tabular}

\footnotetext{
* Syrinx volume is presented as a percentage of whole spinal cord volume for the corresponding segment of cord.
} 

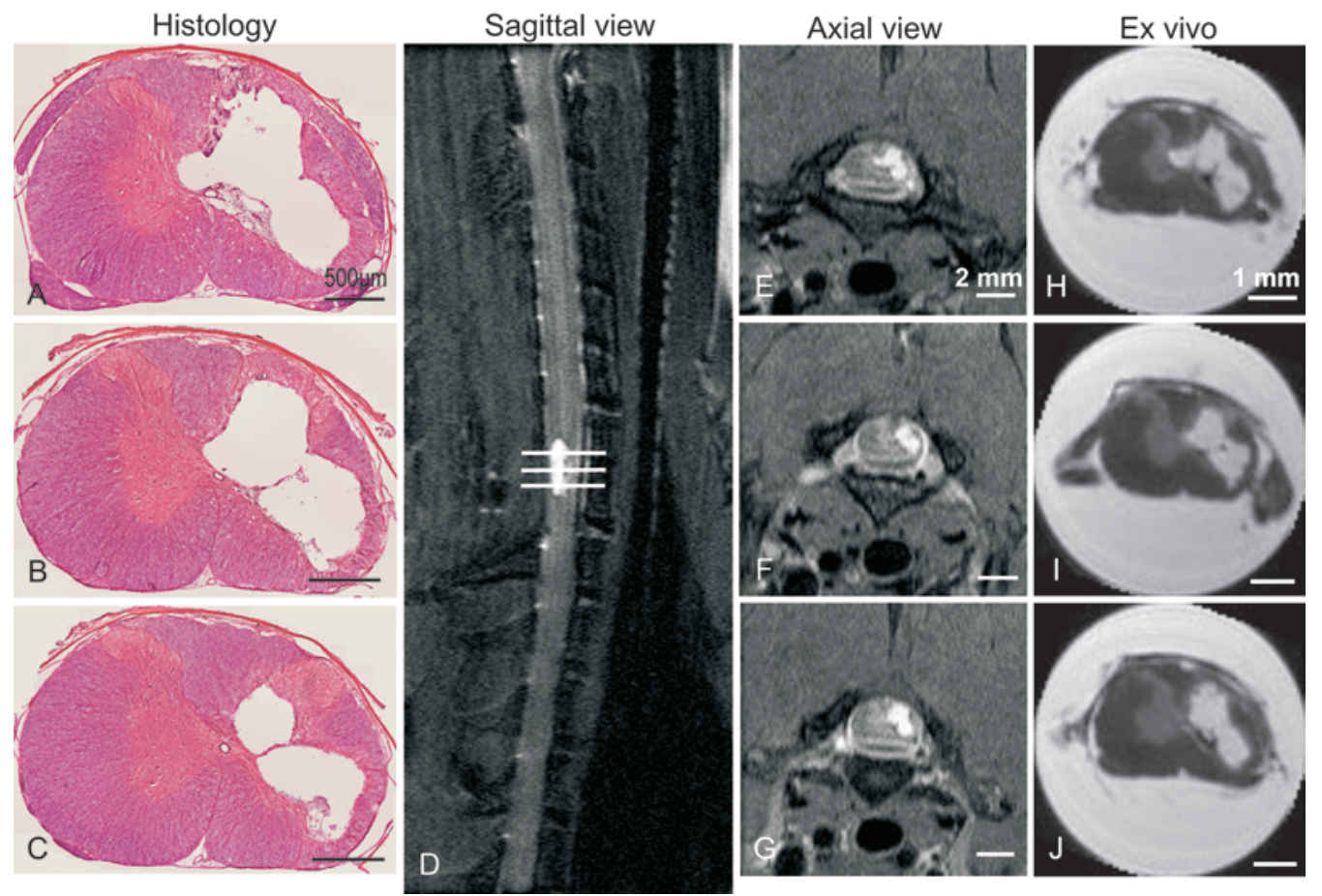

FIG. 2. High-resolution sagittal and axial sections of the T2-weighted MRI correlate with histological changes in the rat model of posttraumatic syringomyelia at 10 weeks. A-C: H \& E-stained transverse sections of spinal cord demonstrating a large syrinx. The corresponding in vivo sagittal (D) and axial (E-G) MR images show the syrinx. H-J: The corresponding ex vivo MR images are shown. There is a strong topographical correlation between histopathology $(\mathbf{A}-\mathbf{C})$, in vivo MRI $(\mathbf{E}-\mathbf{G})$, and ex vivo $M R I(H-J)$. Figure is available in color online only.

vivo images. In syrinx animals, kaolin and/or arachnoiditis was evident surrounding the spinal cord in some animals, limited to the dorsal surface (Fig. 2H-J).

\section{Comparison of Histological and MRI Findings}

Signal intensity changes visible on the in vivo MR images could be correlated with structural changes evident histologically. Regions of hyperintensity corresponded to areas of cavitation or tissue loss. Multiple regions of high signal corresponded to multiloculated cavities separated by septa (Fig. 3A and M, B and N, and C and O). Ex vivo MR images provided additional resolution for closer matching to the histology images (Fig. 3A and Q, B and $\mathrm{R}$, and $\mathrm{C}$ and $\mathrm{S}$ ).

The calculated syrinx volume as a percentage of the cord volume from in vivo MRI correlated well with that from histological measurements (Fig. 5) (Pearson correlation coefficient $[\mathrm{r}]=0.93)$. Syrinx length from in vivo MRI and histology also correlated well $(r=0.79)($ Table 2).

\section{Discussion}

This study demonstrates that preclinical high-field MRI is a feasible method for longitudinally tracking syrinx development in a rodent model of syringomyelia, and that syrinx shape and quantitative measurements of syrinx volume and length from MRI images match well with those obtained from traditional histological methods.

The use of a noninvasive technique for evaluation of syrinx size and location has great appeal from a research perspective. The observation of the injured spinal cord in vivo enables the fragile parenchyma to remain intact for further observation and analysis. Histological analysis also has its limitations, with a previous study noting that estimations of cavity size based on histological sections can result in an overestimation of size because cellular debris, macrophages, and hemorrhagic tissue are sometimes lost through processing. ${ }^{23}$ Accurate calculation of syrinx volume noninvasively would enable syrinx volume to be tracked longitudinally in live animals. This enhances our capacity to study pathological processes and the effects of therapies in vivo. Replacing histological methods with high-resolution T2-weighted MRI would also decrease the number of animals needed for any given study, as changes can be monitored over time rather than requiring multiple animals to be killed at each time point. Sagittal and axial observation of syrinx changes simultaneously can provide further information in terms of following how the syrinx may change progressively from edema and hemorrhage to astroglial proliferation, formation of a cavity, and also multiloculated parenchymal cavities. Ex vivo MRI as an adjunct to in vivo imaging can provide higher-resolution images because it is not subject to motion artifacts caused by respiratory, cardiac, or CSF pulsations that can interfere with in vivo imaging. In addition, longer acquisition periods can be used without risk of anesthetic-related complications. In this study, the smaller radiofrequency coil and smaller slice thickness also resulted in higherquality images with ex vivo imaging.

To the best of our knowledge, this is the first study to 


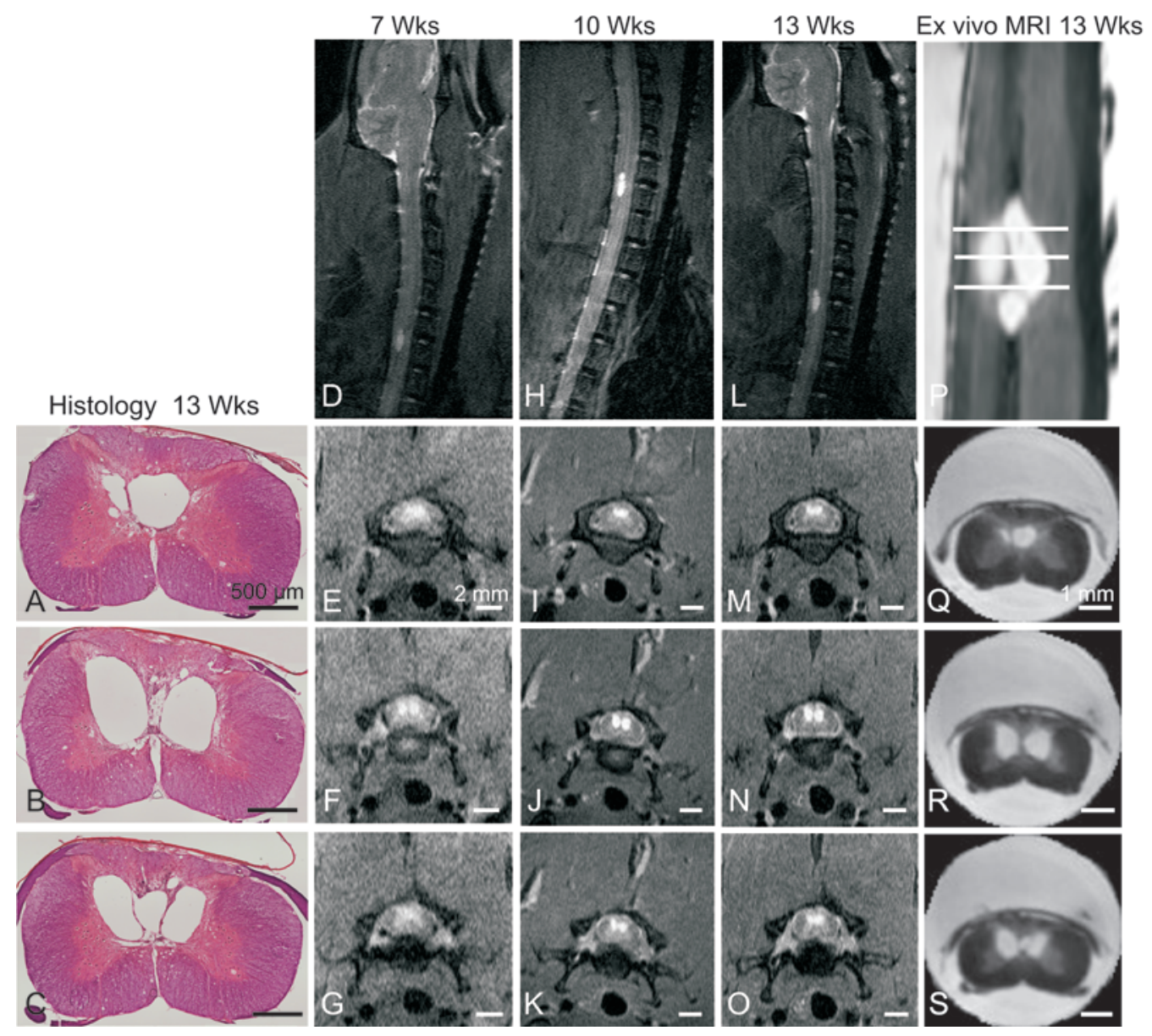

FIG. 3. High-resolution T2-weighted in vivo and ex vivo MR images correlate with histological changes in a rat model of posttraumatic syringomyelia. Representative images are from an animal killed 13 weeks after syrinx induction. Serial in vivo MR images at 7 (D, E-G), $10(\mathbf{H}, \mathbf{I}-\mathbf{K})$, and 13 weeks (L, M-O). Histological sections (A-C) and ex vivo MR images (P-S) show the syrinx cavity 13 weeks after syrinx induction. Figure is available in color online only.

evaluate and quantify syrinx volume in vivo using highfield MRI in a rodent model of posttraumatic syringomyelia. A case study by Bilgen et al. followed the progression of posttraumatic syringomyelia using a 9.4-T MRI scanner; however, this study involved just 1 animal and did not quantify syrinx size. ${ }^{4}$

In this study, the shape of the syrinx as seen on the T2weighted MR images correlated well with the corresponding histological sections. Well-delineated, hyperintense regions on MRI correlated with cavities on histological sections. Multiple high-signal areas corresponded to multiloculated cavities divided by septa, and regions of hyperintensity that were more diffuse or not well defined represented cavities containing cellular debris or edematous tissue. In the longitudinal study, there was a trend toward a progressive increase in the hyperintense region after tissue damage (data not shown); however, this was not significant. It is possible that this is the result of the relatively large partial-volume effect due to the relatively thick slices that were necessary for the in vivo scans. It is also likely that volume trends did not reach significance because of the relatively small sample size. However, as the aim of this study was to determine whether MRI could be used to calculate syrinx size, and the same trend was observed in both MRI and histology, the numbers used were sufficient to achieve our objectives.

In vivo T2-weighted MRI does have its limitations, however. The small diameter of the rat spinal cord requires MRI techniques that can differentiate and characterize small structures inside the cord without requiring an excessively long scan time. This is made more difficult by motion artifacts arising from cardiac, respiratory, and CSF pulsations during image acquisition..$^{14,23}$ We found that the use of moderate anesthesia can help minimize deep and gasping breaths. Cardiac and/or respiratory gating could further minimize motion artifacts at the cost of significantly longer scan times. Longer acquisition times can provide better image quality or higher resolution; however, these benefits must be traded off against the higher risk of complications for animals because of longer periods of anesthesia, as well as the higher cost of imaging time. A limitation of MRI as a tool to study posttraumatic syringomyelia in rats is the available space in which to place animals within the magnet. Chronic studies using rodents older than 3 months may prove difficult because of the size of the animals. It has also been reported that pathologies such as edema and Wallerian degeneration that result in changes in signal intensity on MRI can be difficult to dif- 


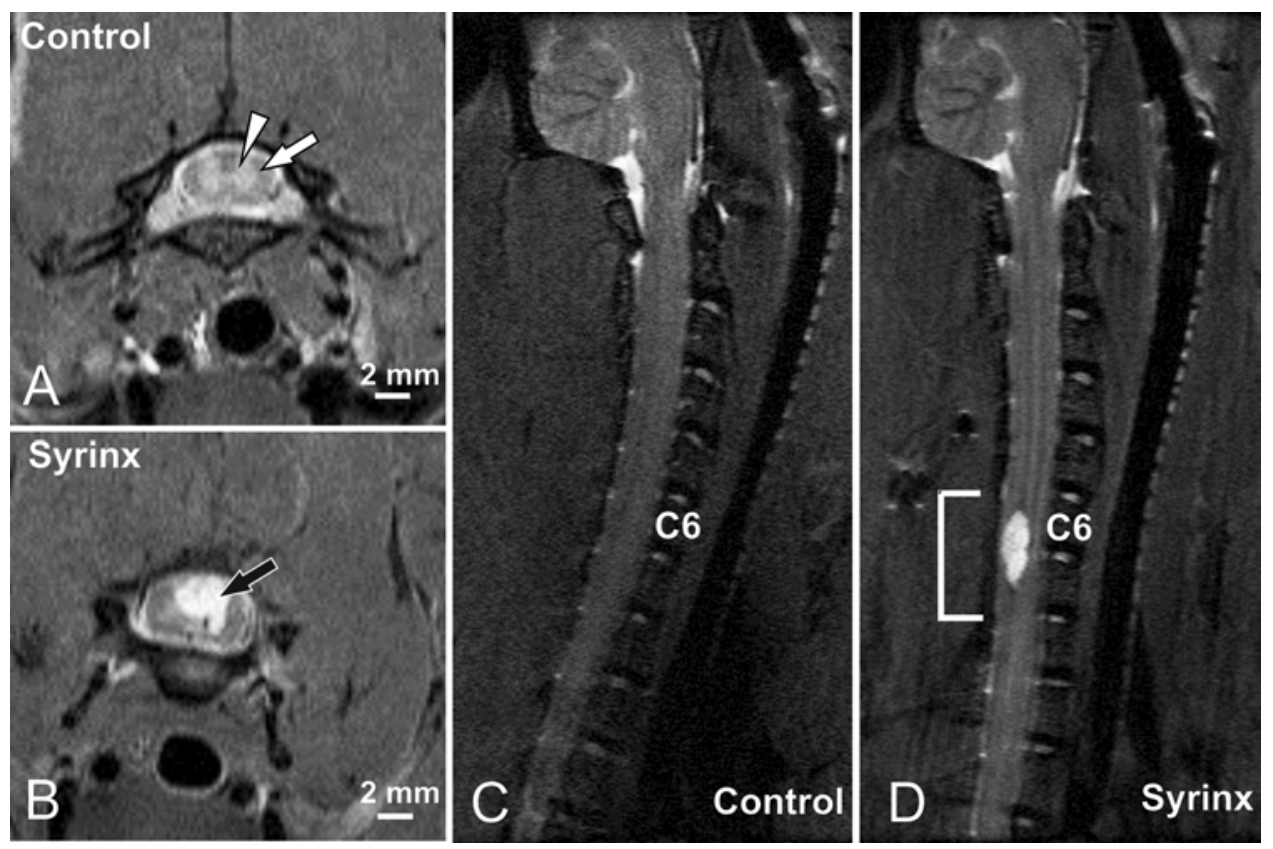

FIG. 4. Representative axial (A and $B$ ) and sagittal (C and D) T2-weighted MR images of rat spinal cord from control ( $A$ and $C)$ and syrinx (B and $\mathbf{D})$ animals, demonstrating white matter (white arrow, $A$ ), gray matter (white arrowhead, A), and syrinx cavity (black arrow, B).

ferentiate, ${ }^{2}$ and white matter tracts cannot be visualized using conventional MRI techniques. ${ }^{20} \mathrm{~A}$ study using in vivo diffusion tensor 9.4-T MRI to study rats after spinal cord injury reported that T2-weighted MRI slightly underestimated spinal damage when compared with histological samples; the authors suggested that diffusion tensor imaging (DTI) provides better quantification of lesion areas. ${ }^{12}$

DTI is capable of detecting changes in the spinal cord

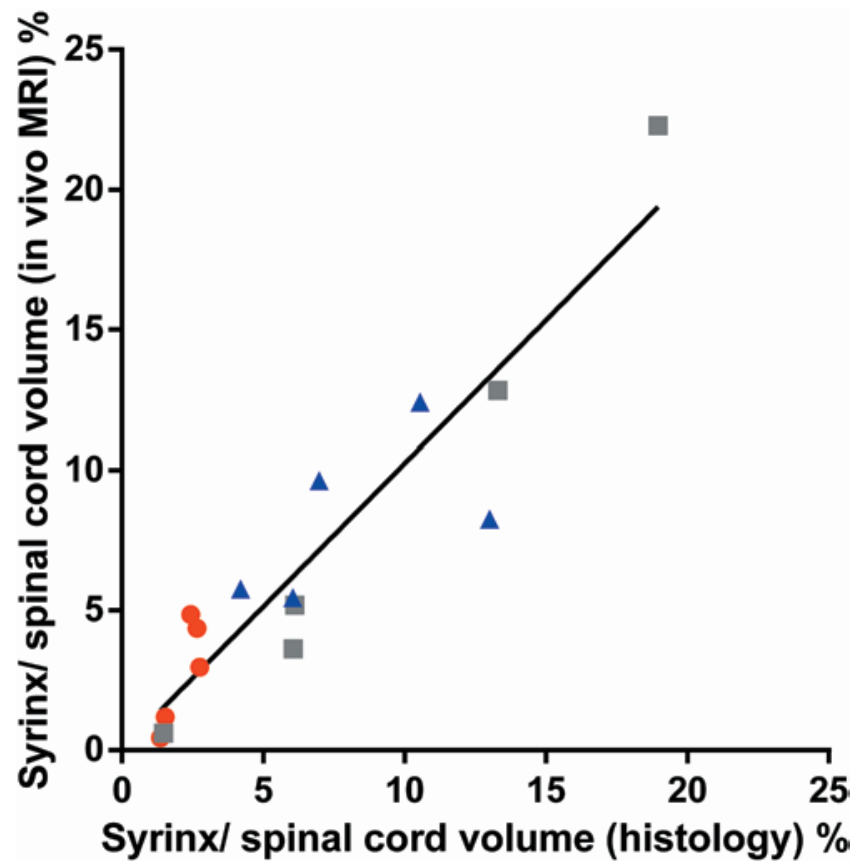

FIG. 5. Comparison between the syrinx volumes as measured by histological studies and in vivo MR images $(r=0.93)$. Figure is available in color online only. that conventional MRI cannot. Several studies have reported a correlation between traditional histological measurements and DTI measurements in the injured rat spinal cord from acute through to the chronic stages of the condition. ${ }^{12,18}$ DTI has been used to observe acute changes such as edema, hemorrhage, and axonal degeneration, in addition to secondary changes such as extension of degeneration and cavity formation within the cord. ${ }^{12}$ Further investigation of our rat model of posttraumatic syringomyelia with DTI may prove useful. However, spatial resolution is much less with DTI, and diffusion methods in general suffer from very low signal, necessitating substantially longer measurement times than T1- and T2- weighted MR images. For both T1-weighted and T2-weighted anatomical imaging and diffusion methods, such limitations could be overcome by technical means: for local detection surface coils could be used. A better solution for overall improvement of image quality would be the use of phased array coils, which would facilitate acceleration methods such as parallel imaging. However, such coils come at a substantial cost and need to be justified by extensive studies.

This study demonstrated the utility of T2-weighted MRI to determine syrinx volume and length. Generally, only animal studies enable direct correlation between in vivo MRI data with postmortem histological findings, which may add to the interpretation of MRI data in human studies.

\section{Conclusions}

This study demonstrates that high-field T2-weighted MRI can be used in place of histological methods to measure cyst size and visualize cyst morphology in rodent models of syringomyelia. This could enhance our capacity to study pathological processes and the effects of therapies in vivo. 


\section{Acknowledgments}

We thank the Biological Research Imaging Laboratory located at the Lowy Cancer Research Centre, University of New South Wales, for allowing us access to their 9.4-T MRI system.

This work was funded by a Column of Hope Research Grant and a Macquarie University New Staff Grant. Dr. Najafi was supported by a scholarship from the Australian School of Advanced Medicine and Macquarie University. Dr. Bilston is supported by an NHMRC senior research fellowship.

\section{References}

1. Abel TJ, Howard MA III, Menezes A: Syringomyelia and spinal arachnoiditis resulting from aneurysmal subarachnoid hemorrhage: Report of two cases and review of the literature. J Craniovertebr Junction Spine 5:47-51, 2014

2. Becerra JL, Puckett WR, Hiester ED, Quencer RM, Marcillo AE, Post MJ, et al: MR-pathologic comparisons of wallerian degeneration in spinal cord injury. AJNR Am J Neuroradiol 16:125-133, 1995

3. Behr VC, Weber T, Neuberger T, Vroemen M, Weidner N, Bogdahn U, et al: High-resolution MR imaging of the rat spinal cord in vivo in a wide-bore magnet at 17.6 Tesla. MAGMA 17:353-358, 2004

4. Bilgen M, Rumboldt Z: Neuronal and vascular biomarkers in syringomyelia: investigations using longitudinal MRI. Biomarkers Med 2:113-124, 2008

5. Bozzo A, Marcoux J, Radhakrishna M, Pelletier J, Goulet B: The role of magnetic resonance imaging in the management of acute spinal cord injury. J Neurotrauma 28:1401-1411, 2011

6. Brewer KL, Lee JW, Downs H, Oaklander AL, Yezierski RP: Dermatomal scratching after intramedullary quisqualate injection: correlation with cutaneous denervation. J Pain 9:999-1005, 2008

7. Brodbelt AR, Stoodley MA, Watling A, Rogan C, Tu J, Brown CJ, et al: The role of excitotoxic injury in post-traumatic syringomyelia. J Neurotrauma 20:883-893, 2003

8. Brodbelt AR, Stoodley MA, Watling AM, Tu J, Burke S, Jones NR: Altered subarachnoid space compliance and fluid flow in an animal model of posttraumatic syringomyelia. Spine (Phila Pa 1976) 28:E413-E419, 2003

9. Brodbelt AR, Stoodley MA, Watling AM, Tu J, Jones NR: Fluid flow in an animal model of post-traumatic syringomyelia. Eur Spine J 12:300-306, 2003

10. Burchiel K: Neurosurgery: diagnosis and surgical management of cervical syringomyelia. West J Med 145:84-85, 1986

11. el Masry WS, Biyani A: Incidence, management, and outcome of post-traumatic syringomyelia. In memory of Mr Bernard Williams. J Neurol Neurosurg Psychiatry 60:141-146, 1996

12. Ellingson BM, Schmit BD, Kurpad SN: Lesion growth and degeneration patterns measured using diffusion tensor 9.4-T magnetic resonance imaging in rat spinal cord injury. J Neurosurg Spine 13:181-192, 2010

13. Fedorov A, Beichel R, Kalpathy-Cramer J, Finet J, FillionRobin JC, Pujol S, et al: 3D Slicer as an image computing platform for the Quantitative Imaging Network. Magn Reson Imaging 30:1323-1341, 2012

14. Fenyes DA, Narayana PA: In vivo diffusion tensor imaging of rat spinal cord with echo planar imaging. Magn Reson Med 42:300-306, 1999

15. Fernández AA, Guerrero AI, Martínez MI, Vázquez ME, Fernández JB, Chesa i Octavio E, et al: Malformations of the craniocervical junction (Chiari type I and syringomyelia: classification, diagnosis and treatment). BMC Musculoskelet Disord 10 (Suppl 1):S1, 2009

16. Hemley SJ, Bilston LE, Cheng S, Chan JN, Stoodley MA: Aquaporin-4 expression in post-traumatic syringomyelia. J Neurotrauma 30:1457-1467, 2013

17. Ko HY, Kim W, Kim SY, Shin MJ, Cha YS, Chang JH, et al: Factors associated with early onset post-traumatic syringomyelia. Spinal Cord 50:695-698, 2012

18. Loy DN, Kim JH, Xie M, Schmidt RE, Trinkaus K, Song SK: Diffusion tensor imaging predicts hyperacute spinal cord injury severity. J Neurotrauma 24:979-990, 2007

19. Schurch B, Wichmann W, Rossier AB: Post-traumatic syringomyelia (cystic myelopathy): a prospective study of 449 patients with spinal cord injury. J Neurol Neurosurg Psychiatry 60:61-67, 1996

20. Schwartz ED, Chin CL, Takahashi M, Hwang SN, Hackney DB: Diffusion-weighted imaging of the spinal cord. Neuroimaging Clin N Am 12:125-146, 2002

21. Schwartz ED, Yezierski RP, Pattany PM, Quencer RM, Weaver RG: Diffusion-weighted MR imaging in a rat model of syringomyelia after excitotoxic spinal cord injury. AJNR Am J Neuroradiol 20:1422-1428, 1999

22. Ventureyra EC, Aziz HA, Vassilyadi M: The role of cine flow MRI in children with Chiari I malformation. Childs Nerv Syst 19:109-113, 2003

23. Weber T, Vroemen M, Behr V, Neuberger T, Jakob P, Haase A, et al: In vivo high-resolution MR imaging of neuropathologic changes in the injured rat spinal cord. AJNR Am J Neuroradiol 27:598-604, 2006

24. Wong JHY, Song X, Hemley SJ, Bilston LE, Cheng S, Stoodley MA: Direct-trauma model of posttraumatic syringomyelia with a computer-controlled motorized spinal cord impactor. J Neurosurg Spine: [epub ahead of print January 29, 2016. DOI: 10.3171/2015.10.SPINE15742]

\section{Disclosures}

The authors report no conflict of interest concerning the materials or methods used in this study or the findings specified in this paper.

\section{Supplemental Information \\ Previous Presentations}

Portions of this work were presented in abstract form at the 25th Annual Scientific Meeting of the Spine Society of Australia, Brisbane, Australia, 2014.

\section{Author Contributions}

Conception and design: Hemley, Najafi, Bilston, Stoodley. Acquisition of data: Hemley, Najafi, Bilston, Song, Bongers. Analysis and interpretation of data: Hemley, Najafi, Bilston, Song, Bongers. Drafting the article: Hemley, Najafi. Critically revising the article: Hemley, Najafi, Bilston, Stoodley, Cheng. Reviewed submitted version of manuscript: all authors. Statistical analysis: Hemley, Najafi. Study supervision: Hemley, Bilston, Stoodley.

\section{Correspondence}

Sarah J. Hemley, Faculty of Medicine and Health Sciences, F10A, 2 Technology Pl., Macquarie University, NSW 2109, Australia.email: sarah.hemley@mq.edu.au. 